BELIZE

1981

M. Costalis

1a. Nasutitermices sp. Im $2, \%$ from $\log$ furing used as freward. Tavi 4,198 Blme Hole, Belize cMagui)

1B. Termitoside foom 1A. in spee pres.

2. Amiterme ap 2, 7-dean

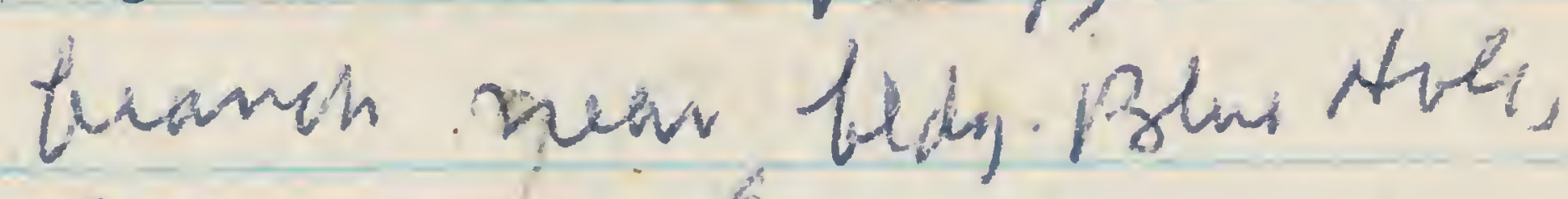
Beling Jumelo, 98

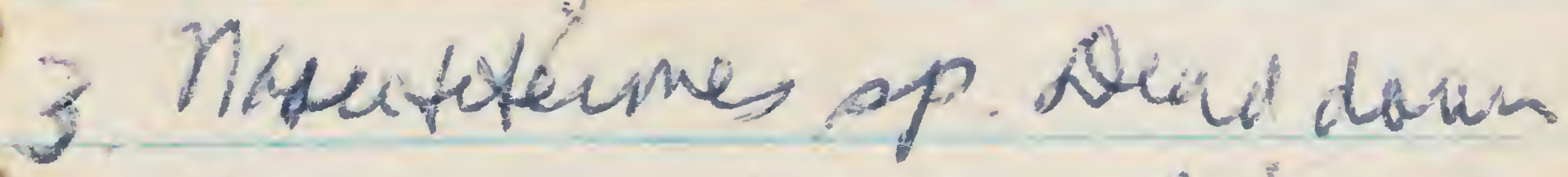
haven, mitrais to bulding Bene thole, Belions Tume 7. 1981 ISV + vanches. Maphes 4. Qmiterma op Jm, 4,7 Jank, 1981 dewn trumen Blui Btole - Beluze 
$r 83$

3)

5. Hefewtermes of 4 \& 8 Jun 6,1981-Doundead bianch nean liveding folut Aor, belize. (Destrised, pacto 6. Heferoteumes ip. June 7, 1981 Stunep, hervily eaten, in grvendBlus zbre, feling 4,7 Snue?

Q. Combat 1: Rauge ant unuing on a thoil Hexparime added, tow disinyted, of 앙 4 attackes and dume of ants
Microedotormes

Compat 2 - Rel army ant is seferotemes os - ants mere dived from triw, Eimites atlached and

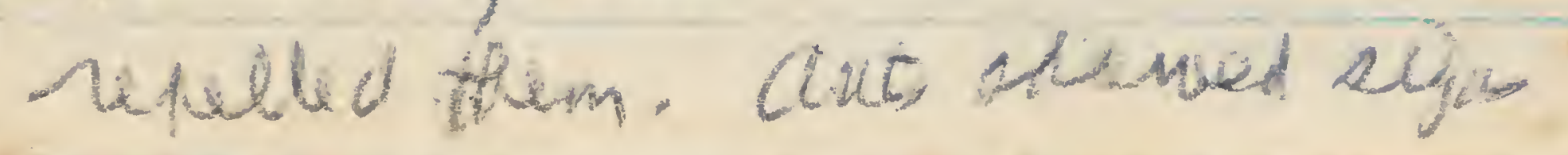




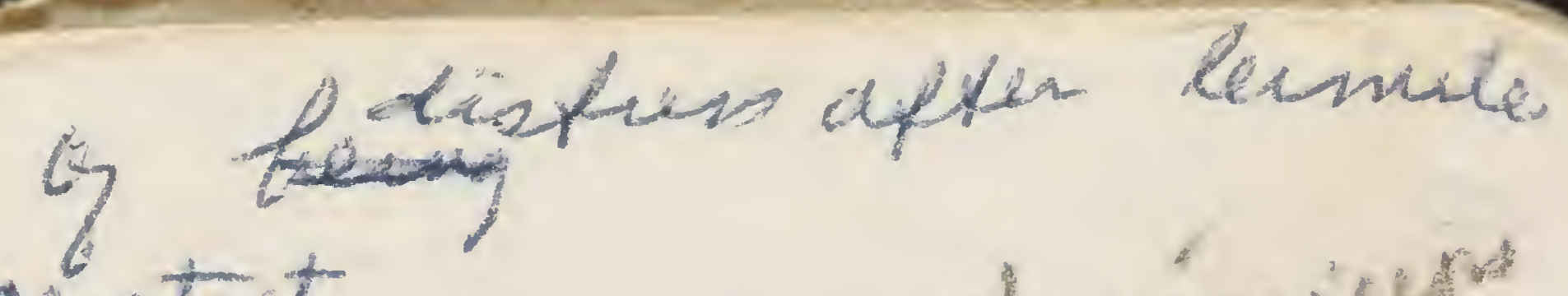
contact.

Combet 3 Ne migielp woramy ant wo nasututermes - tirmile repellid aut eptesturez, Juks 7. Bexile Rav- 18 ne

4

June B Nacutiterme 18 8. Suricolong - 7ever Pout

boside noad topare pras

4004 me name

9. Dead Funch on grouszd Linde road in second-grawth furdering Calumbir Ra. ca 5 me quan

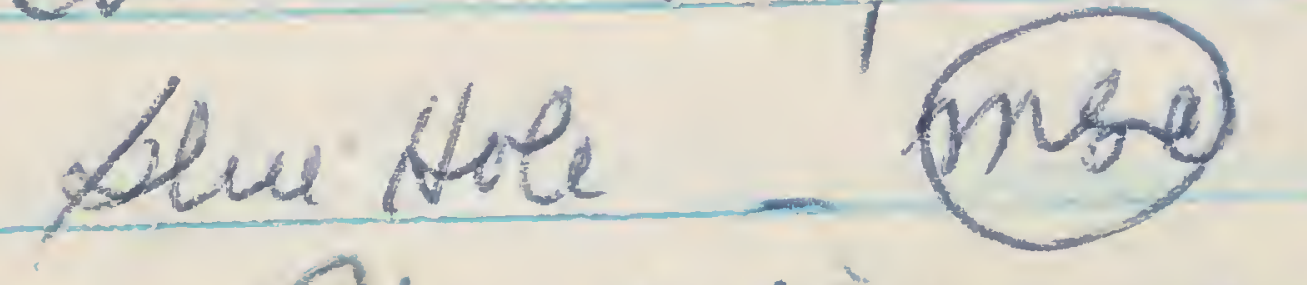
10. Staplatmids eating stray teimites as site 9 neat priking - Nosutterme-m 
11 Janes-Beside rave, secont Gowth, near Store, 6 mi from Blue Hole, Belige. 7, क 4a 2030 - 506070

12. Jume-Buids Rad-cuton neot on palmtal, en 1/2 pr Marregrauns a cami, four plue Holistation, clewed are, leside nud - Se cond-gowth farest nowutionses sphatioe. 1002 in Bepare

Combat is monacis (3ap. gor gers pietures -

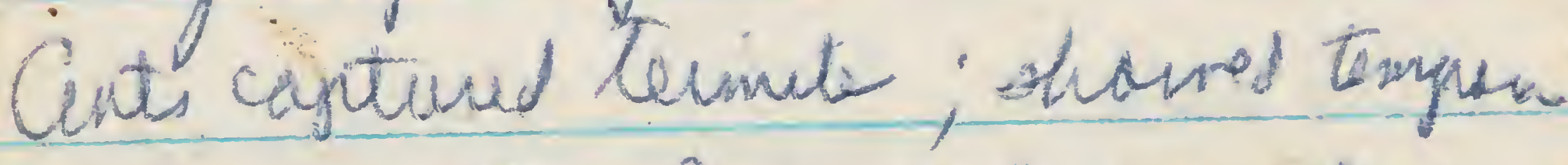
distess apter being pired an, berk ment on to clean, Ghen attach mice letmo b 
Nest metereal with termits in it paaced in midst of Coorbat of 10:55 AM. Tfter

P / hi: 2 en

13. Qumite 75 - prom

leary

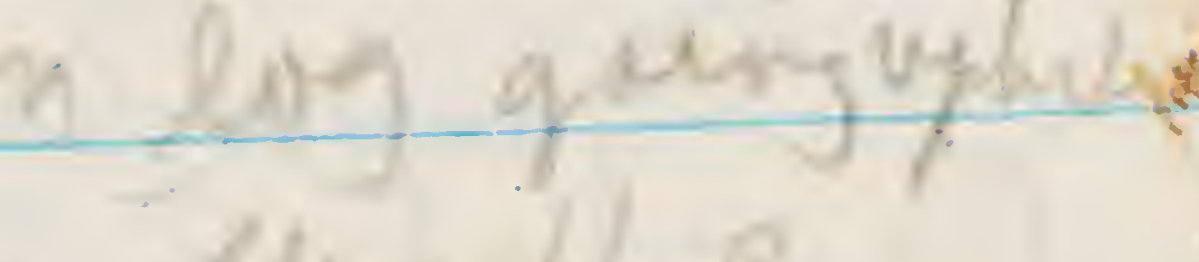

14. To recent, incry

Dearfersych on ground samel

as 13 , funther ut- Mary ano

Encly_-gave 9, 1981-uscin 13 
15. Holkesticrocentumes sp. standing steypu - up hais fromplue Horle dodge latrios Jurea.

16. Nuentutimes ep litoctmilis in larbs T omble, eles fehaves Inmea, $m_{1}$ ores awera Combat $c$ Movacis Buch of wot confainis Ca 30 nasibitam op wabs 4 sordies placed in path of poraging moneses. Cints weu initant repellos creble around termits. Junite retion fiest 
any aut thet agperates, group ste untod apte 30 min. Padies teparater aut leaving urard, later cutuend. 2/ slow, but squiller ants effeptered i̊ caught at le givnory $i$ i e $45 \mathrm{~min}$.

Terminater e / la - surr wos ulionel to euffare 6 aproyd and istalet is plasticens 17 Amiterme sp 2, q 7. Nocon wad near lodge Blus Hot, (70) $2003 \times 1050$

18. Dentotermes of lange everon in rottin by in thíl to falls, blue Hols, beliza 10 Jum 1951 - mse 
16-Continue:-

24 hus leter-survires

- 3 livin, Idex,

2 escaped

Comfint $\# 15-12: 308 \mathrm{~m}$

ex 500 une 10

C. Dermite fron colvong pleney in Monaces traís $12=30$

and suryed araben inmelise aloo predier

Nearly all termites capturs and carried of ly wht within 30 minnites 
19. Coptifume tp unde buk, dead tue - doll Earmen Steiner -Jiene 10, 1981 blue tole, belige

20

R/

Spelluis caned tonglen

N. ephrotale

22 - save a lomáas 21?

arubrio fo funtur doren trail in deal leavo-blaced in tras as bait for predarors

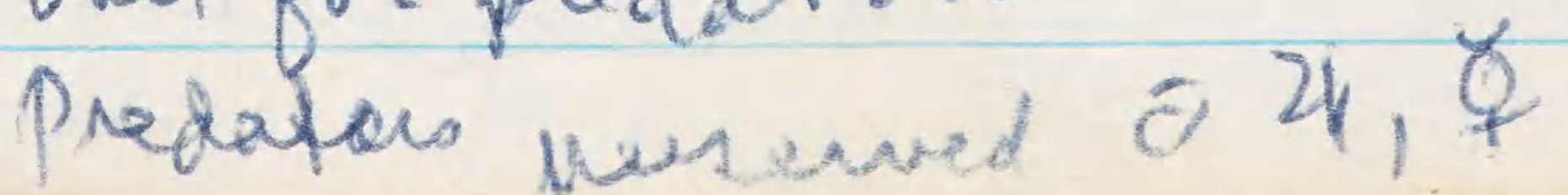


Dewis ant fer dead trees inhalintes by White. Cowstrad Pant

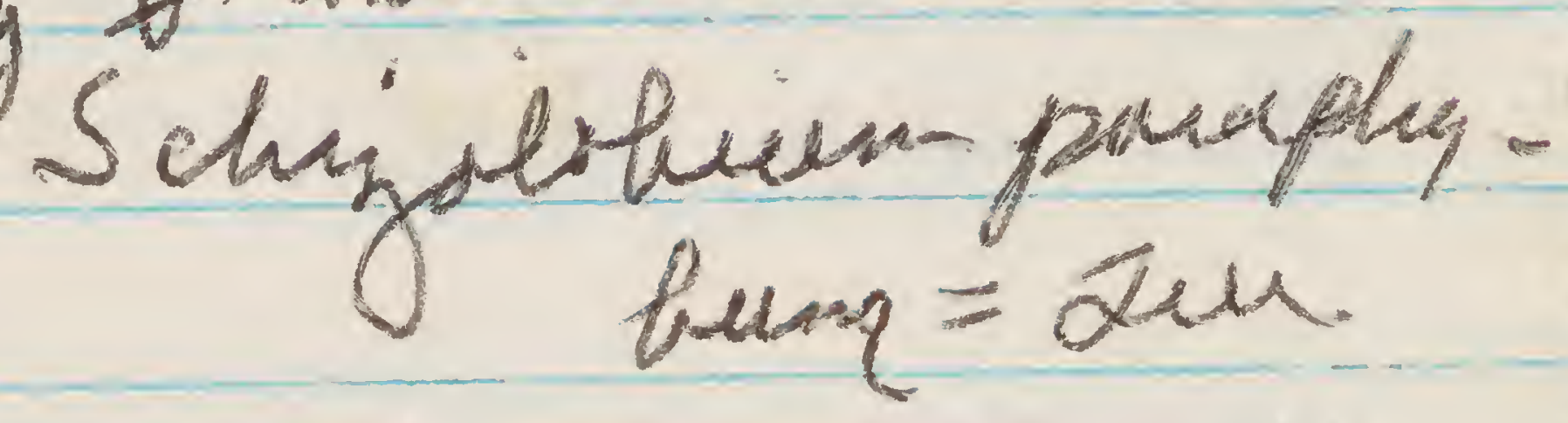
3 neat in oren, dll vin toes wholibeoly and protucted big rese gants. very iverses!

23. Jose 11, 1981 fores/ Surves Station near solamanca-geo beat. Smovi carton nest on arde gorang tres in clensi ivar -

200 te Aeptane; 2, \& vurehes Compes: 100 市, 1002 un feeding haie o manaes $A$ 


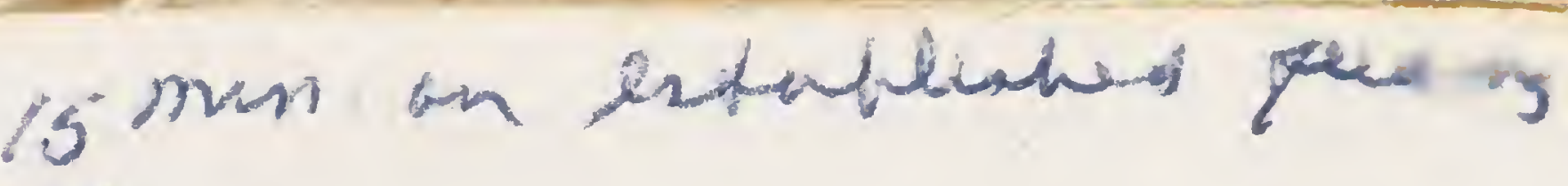
sile.

24. Tum 11- Jovet Servir sta.

cautor neat an kee. 2003 to bepane; ant paratos olserres -

Combet: Monocis" indriados

matrewatarily cupurbed thy 2 fire bus som verver and ottach goupad a isclatio wathes. Caryiar of eary numbers to nest.

25. Lay conplet caton nes eging angulund thind okhaid, Joustsence Sta pelige-Junell - 200 to leypais 
auts ate there speck a despitich $N, 2$, id, guests pres.

26. Anitermes sp. varren Seine Columañ cuerd, belize Jowe 11, 199,

27. Coptatumes op boven $\log m$. funiding, Bue coeh. Jume $12,198 \%$ 28. Nasufiternes sp. eartar kest on tree, ew grouss men beach, Beliem proch klotel-Jume 15 $100^{\circ} 4 / \operatorname{Im}, 4, \%$ 
29. Nasutifums st Alangriga - on tur near Gelicar boch Natel. Carkm nest. $100^{+}$y / Im, 21, \% Suath Hewray, 45 mi so. od dangigig.

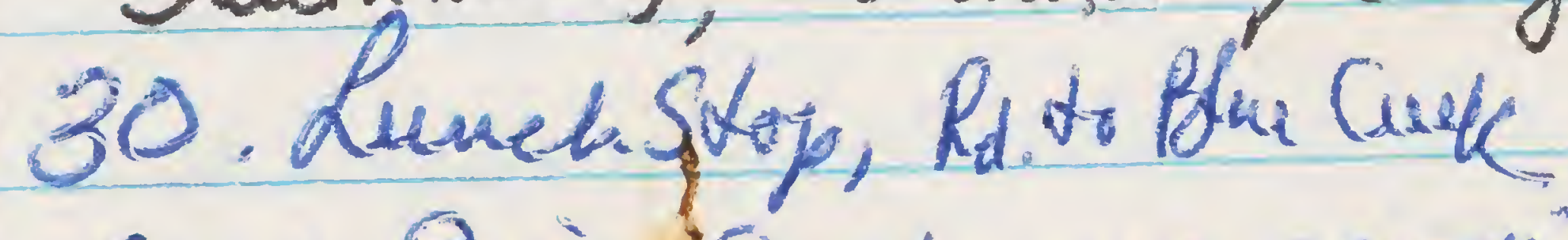

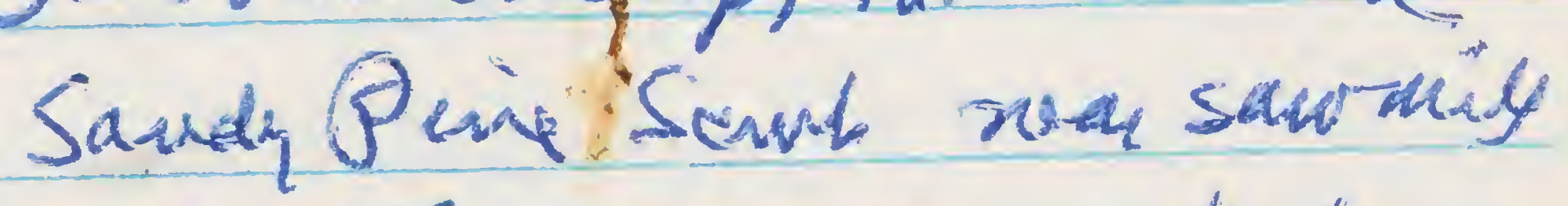
aite-June is, 1981-under bangs $\log$.

31- sam as 30-Amitum as

32. Netartions ote yne ina, deanl fround 33. Srame tata a 32 34. Aurtermes \& foon con chíq-Samesper 

35. Copptotems ep

in parts. herier some

lig-ald coltre o cartong!

lath muss inoids.

same sits, enench sty.

Blu cuex agani-jum 17,

37. Armitermes of. Jum 17, 1981

Rottenling $m$. sheltes Misc.

38. In votter log, same dus as $\# 37-14$

Aeferatermes spo

39- Retten log Juither up

towis - with Amblypygid - 
40. Nasutiterme Cutonnest on side of big ther rear lations-esluicula 8tx. - netwith thin autar exvelop, fuimes univices 1003 ph glesn

It amite -7

42. Koyeumiti. ifaed stunding stury nea shelle, sant day In, $4, N$ Celoneto Lamm

43. Aviderves sp. ants, life cyde 
44. $\operatorname{lag}$ annitum fors $\log \bar{e}$ cexpipils

45. $n$. oetquil (?) foum lage dead log nen Malairs sor, $1 / 2 \mathrm{mi}$. Jow ohelter-Dive cutimy Pon solie sume 17 how N. Glahsifergues

Ho, Qnitermes as. Jume 18,198 down Gionch near bluk hech, 14 mi upriver from ablfew mse. 4, I 



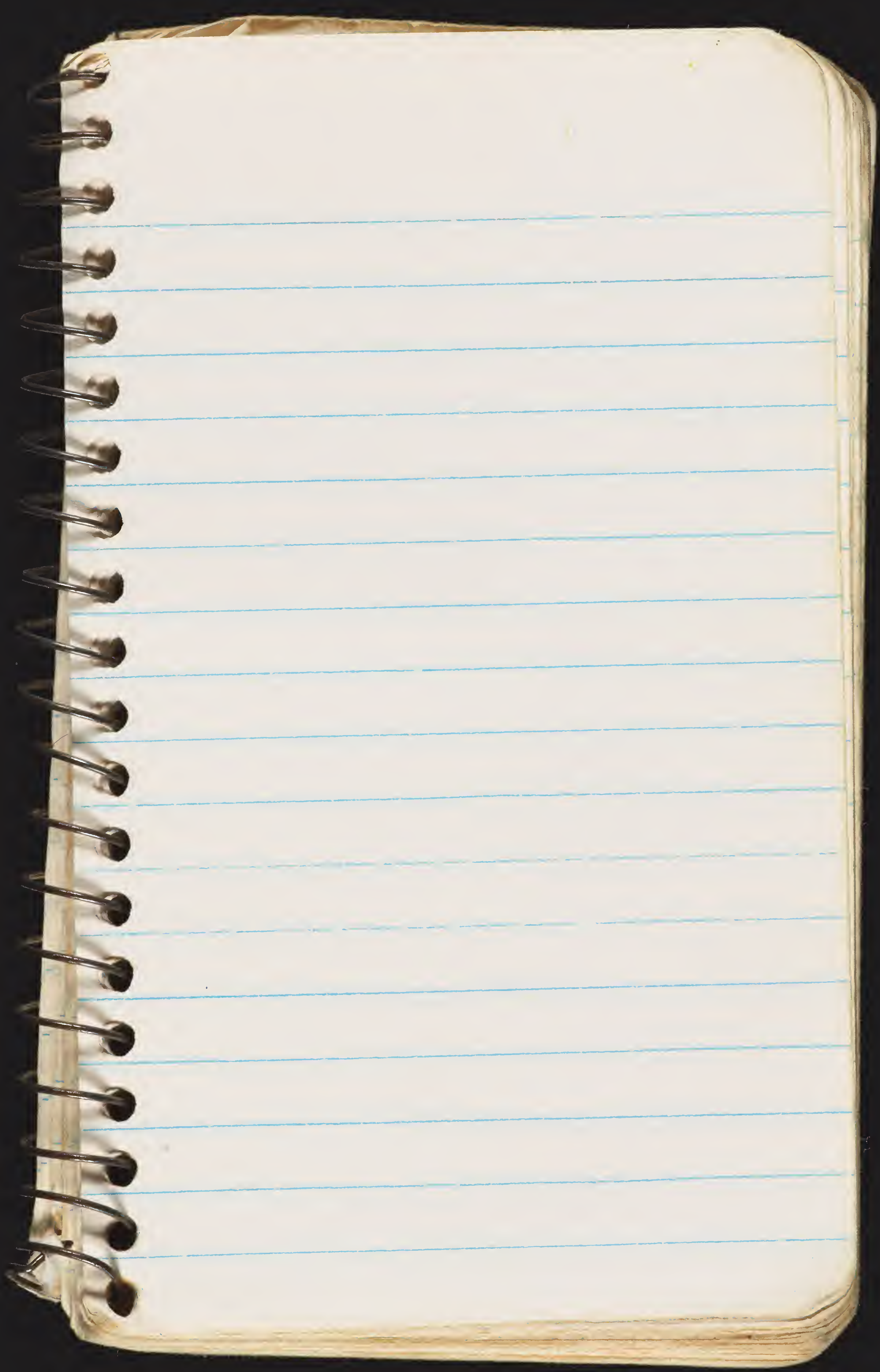





$$
\text { Kay } \begin{array}{r}
357.1989 \\
+989
\end{array}
$$




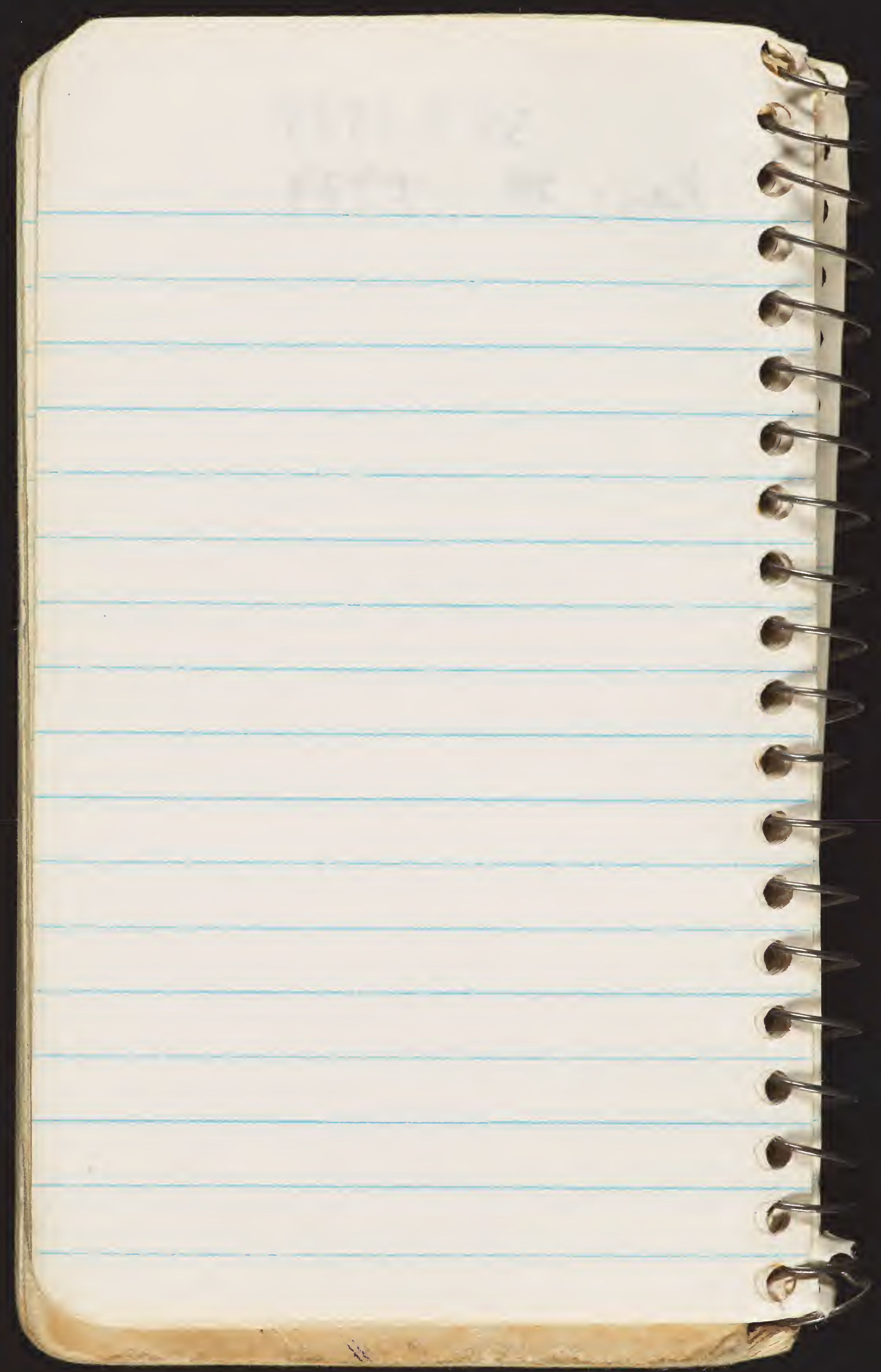




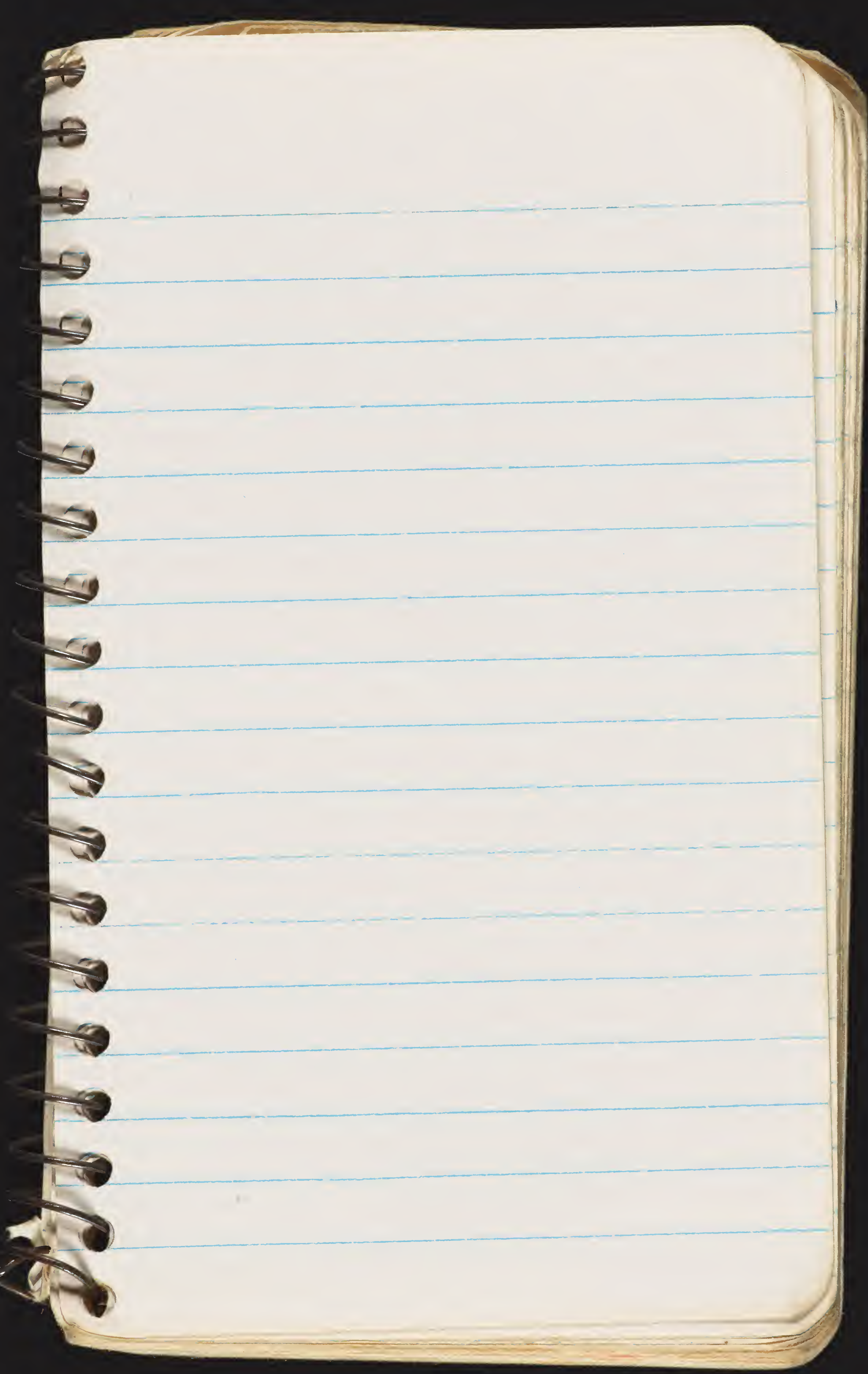




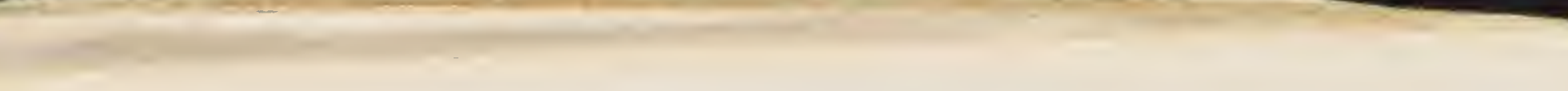

C

(1)

e⿻ 5

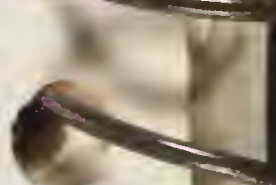

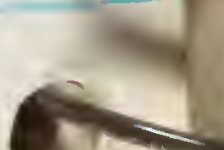

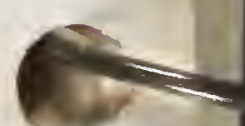

0

6

6

A

$\Rightarrow$

$\infty$

N

$=$

-

E)

SI

$\theta$

8

e.

e)

(4) 


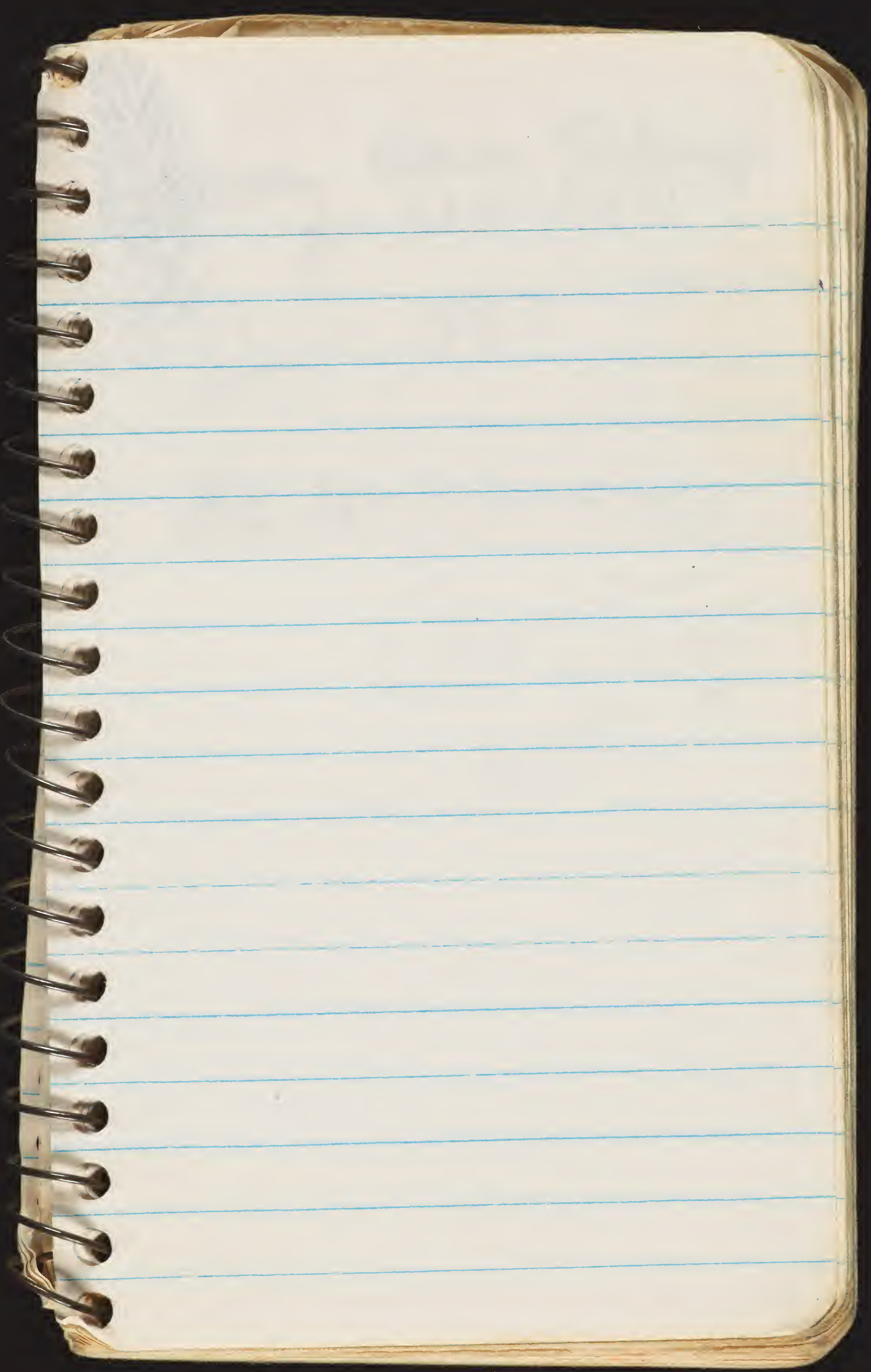



Robenta Rubinsft

Poby -282.3271

$$
\begin{aligned}
& \text { Mare-382-8982 } \\
& \text { HEM-770-5200 }
\end{aligned}
$$

Fnoer seminaw

12:00 owre 1:15 2 not then * 

5. 615 guryameric-2272338

* limenear Ainand $661-42 \times 2$

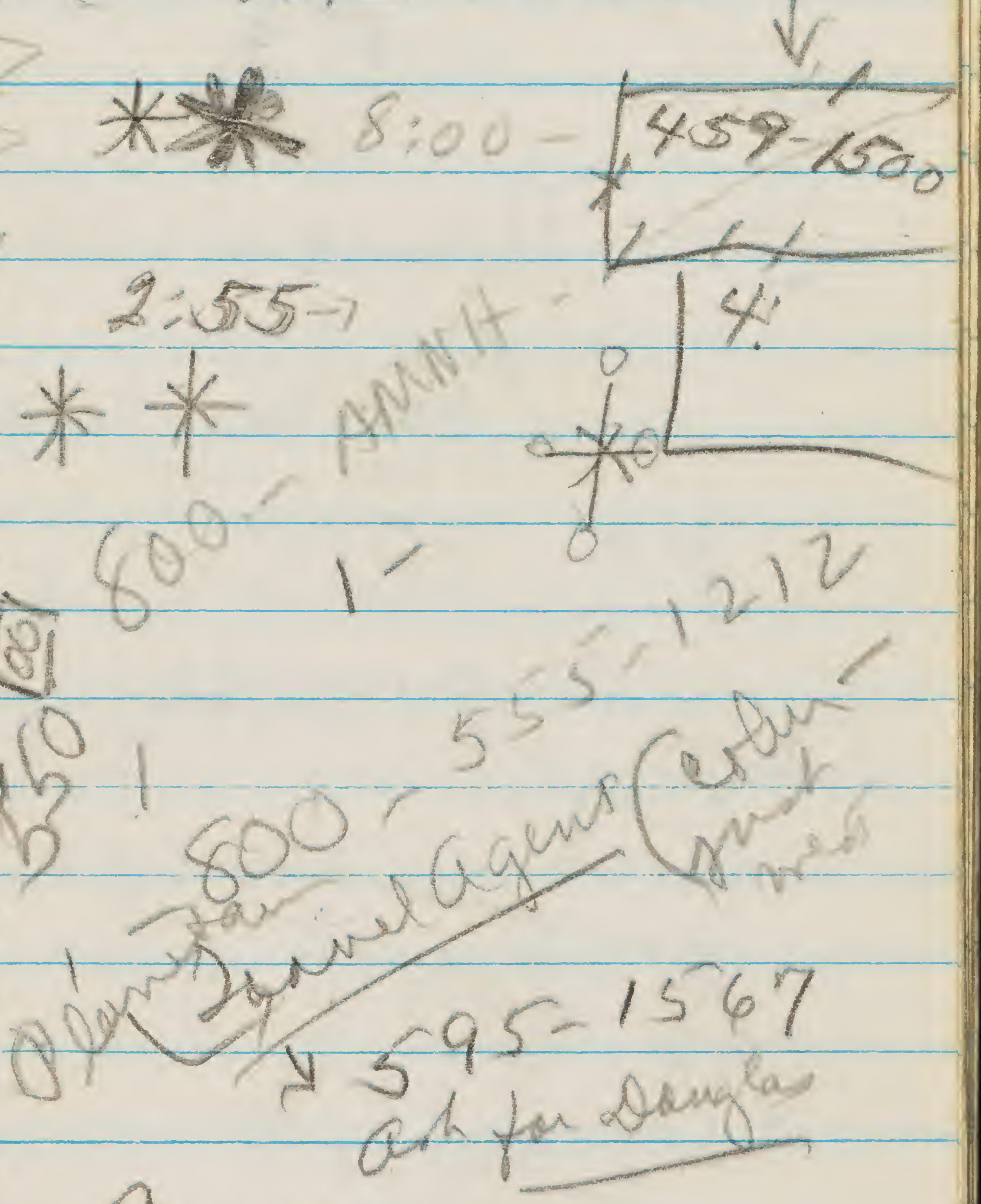

OVISA 

Wel peliz specumas book alese

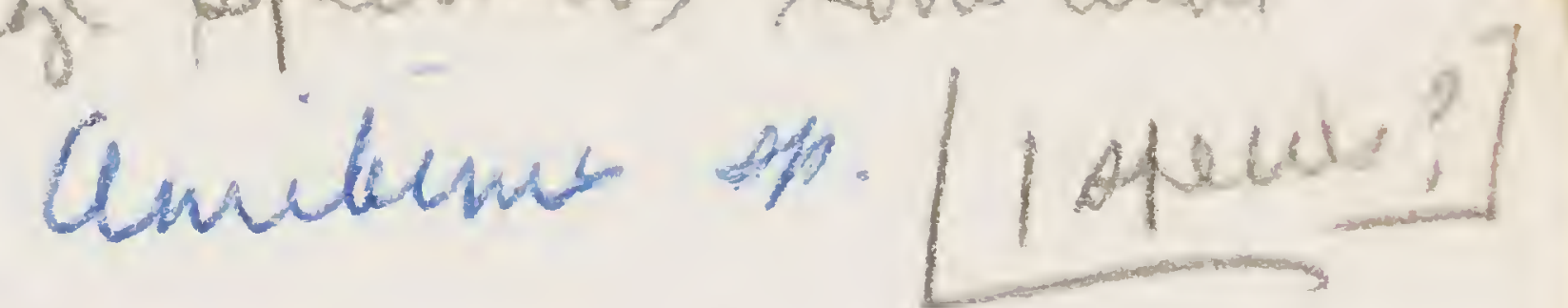

430 foelt mud. 1.72 mas.

H 43 - as atore

\#14- as ation

plus 1 osd feete

12 as afort

+ 31

$46,-1$ 


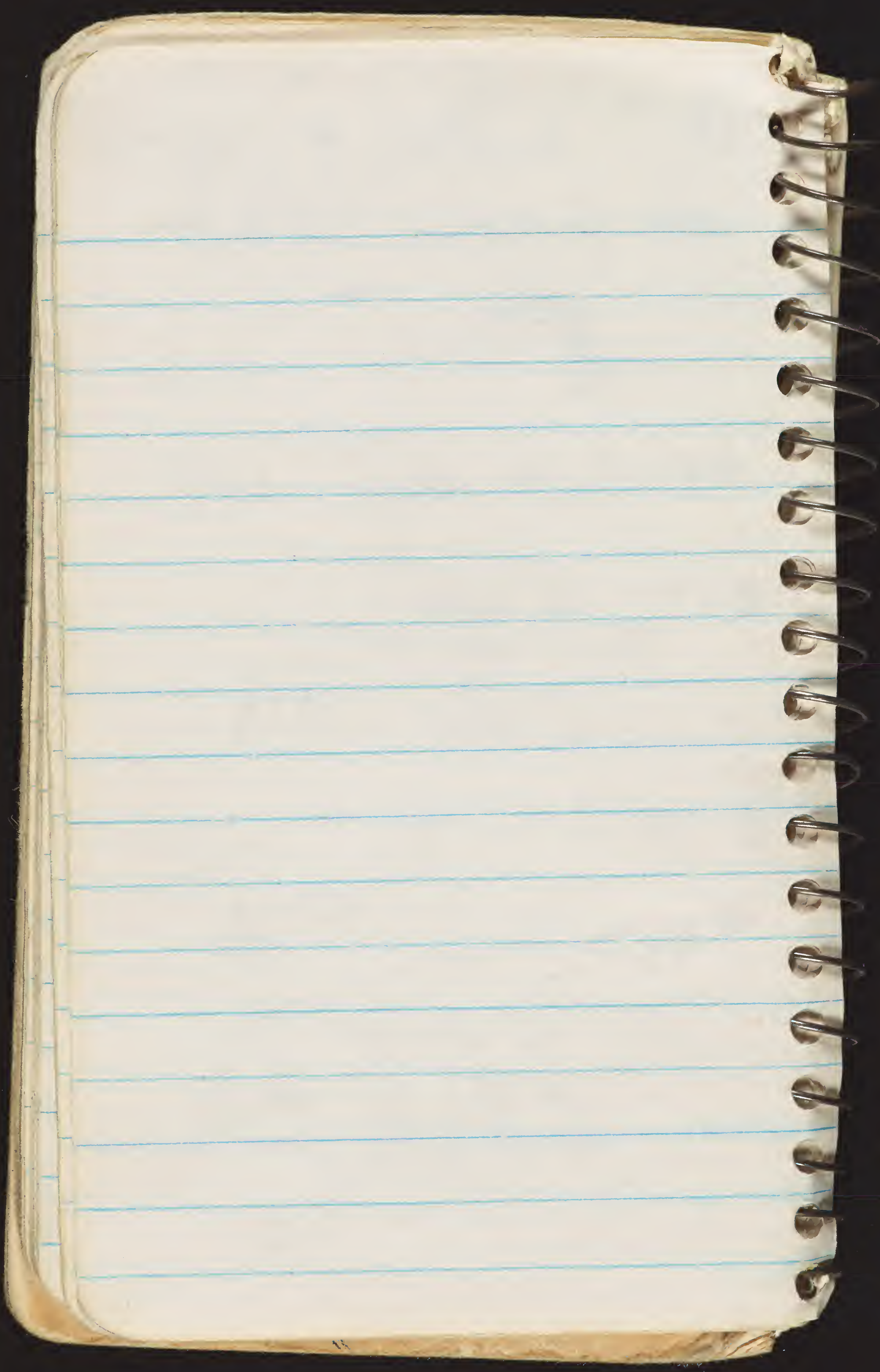




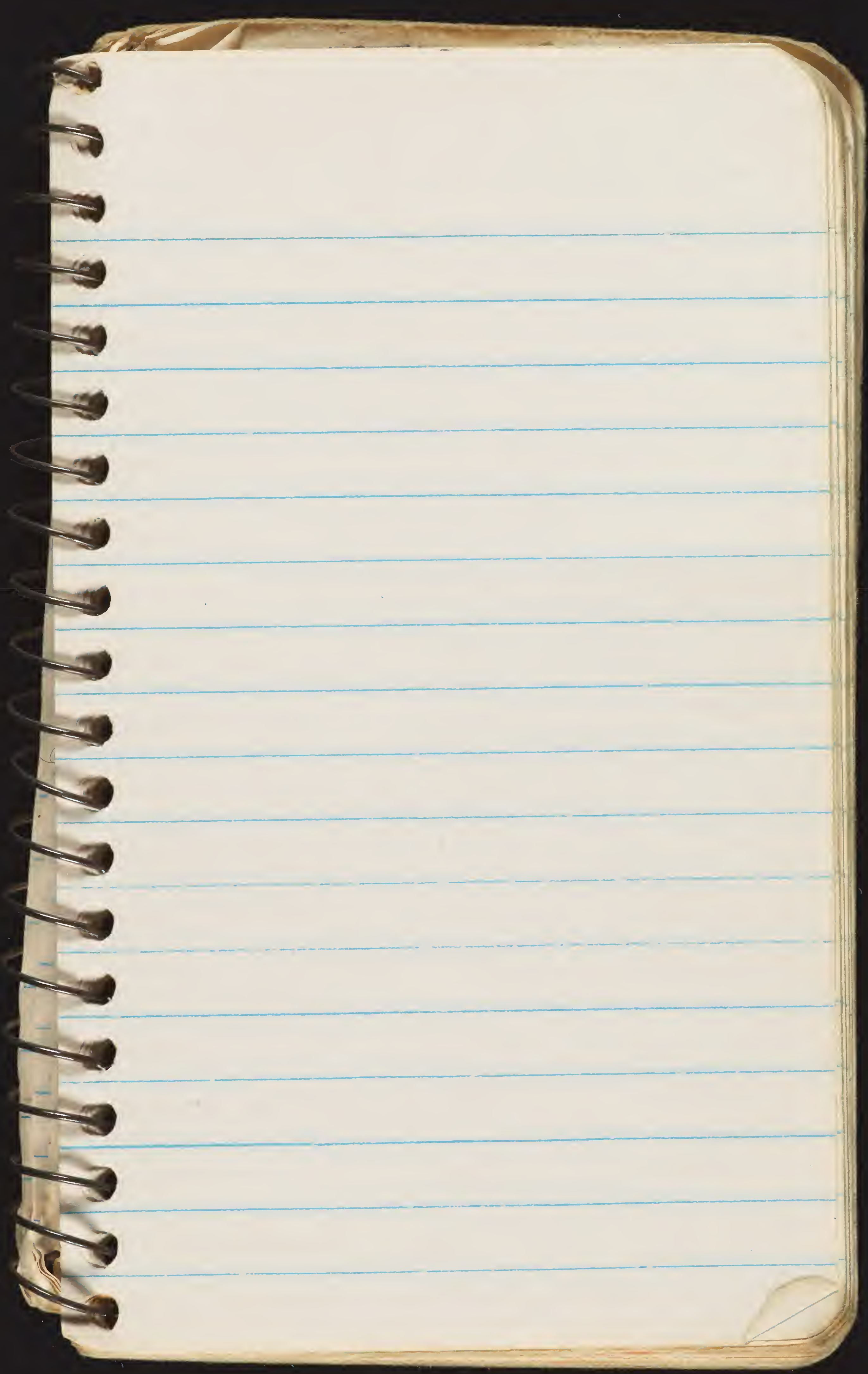



2 woll 89.95

Susen $\times$ Dare thian Harreat Saun -

$$
1-800-848-2649
$$

1 wisa th

$$
\begin{gathered}
4332-0001.0509- \\
8678 \\
\text { cop 1/90 }
\end{gathered}
$$

Sevd To:

20 ancire in ca 2 mees 



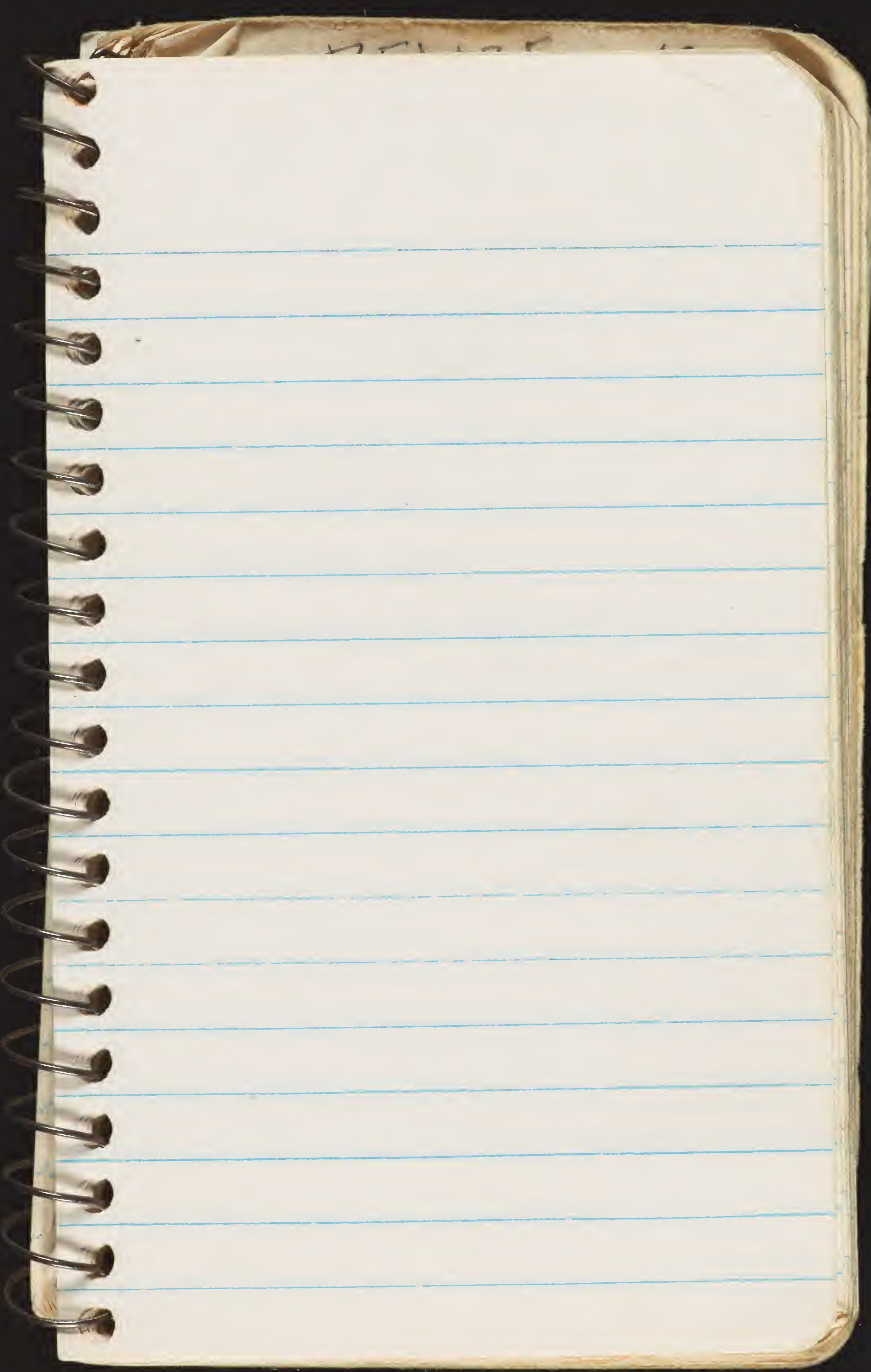





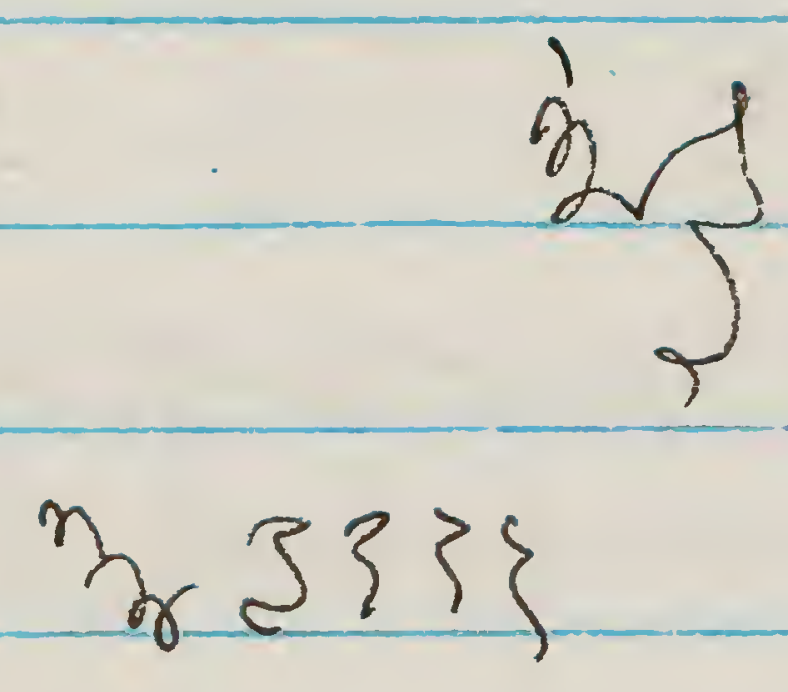

Armitermes albidus

Macracanthorhynchus hirudinaceus

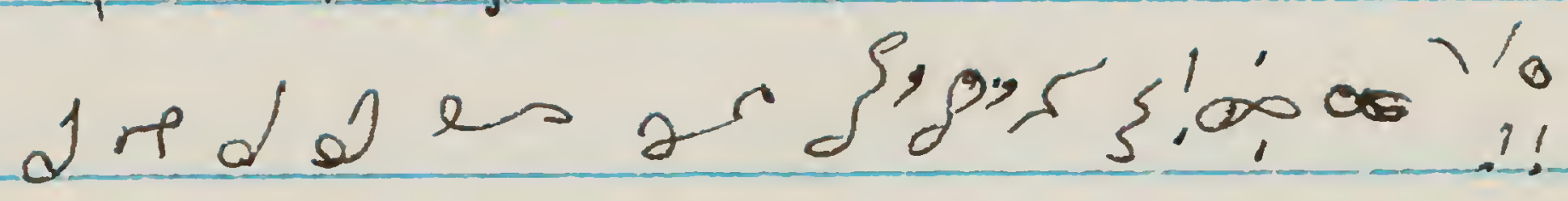




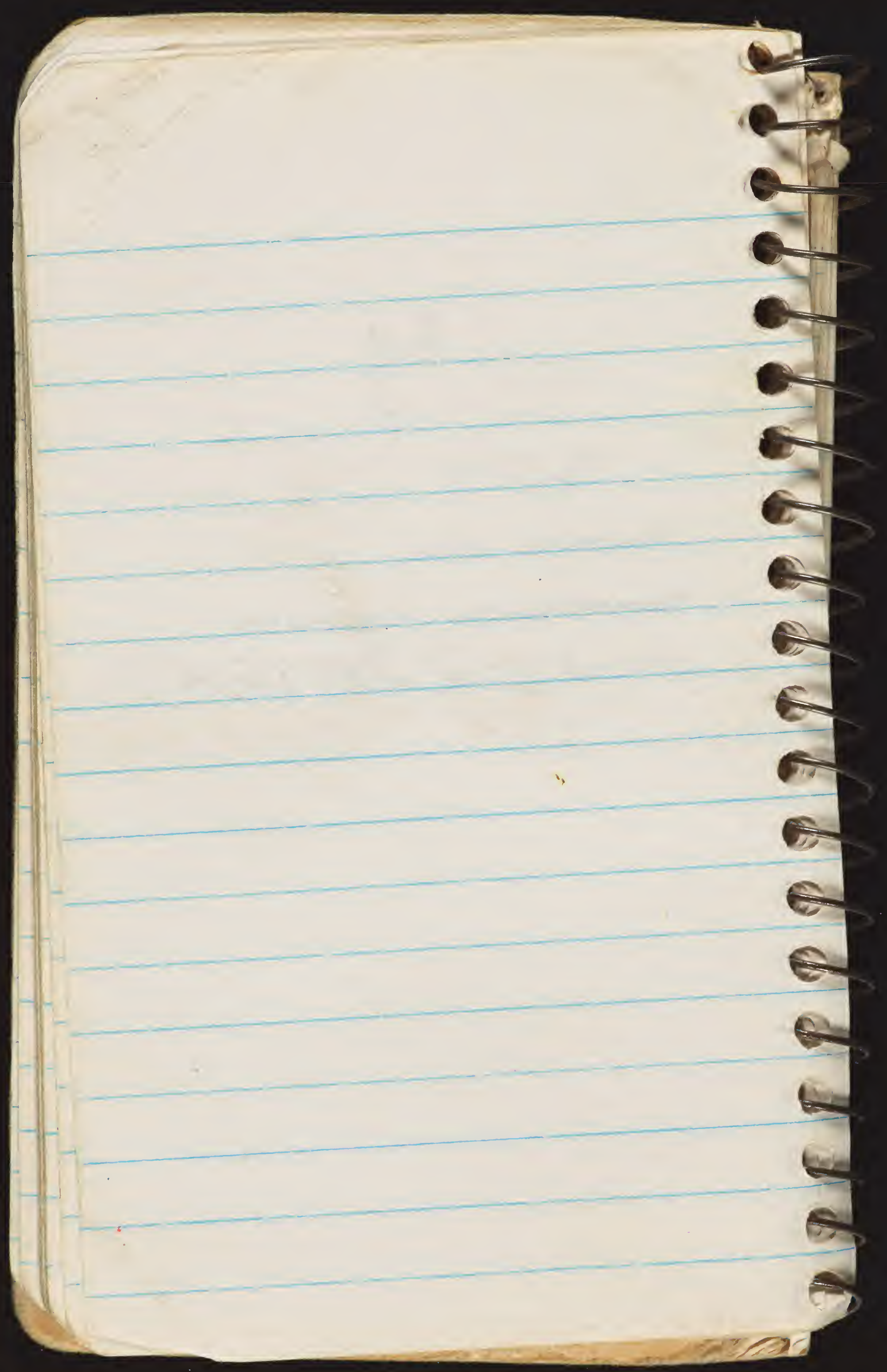


him $\beta$

$$
\text { 333-6684 }
$$




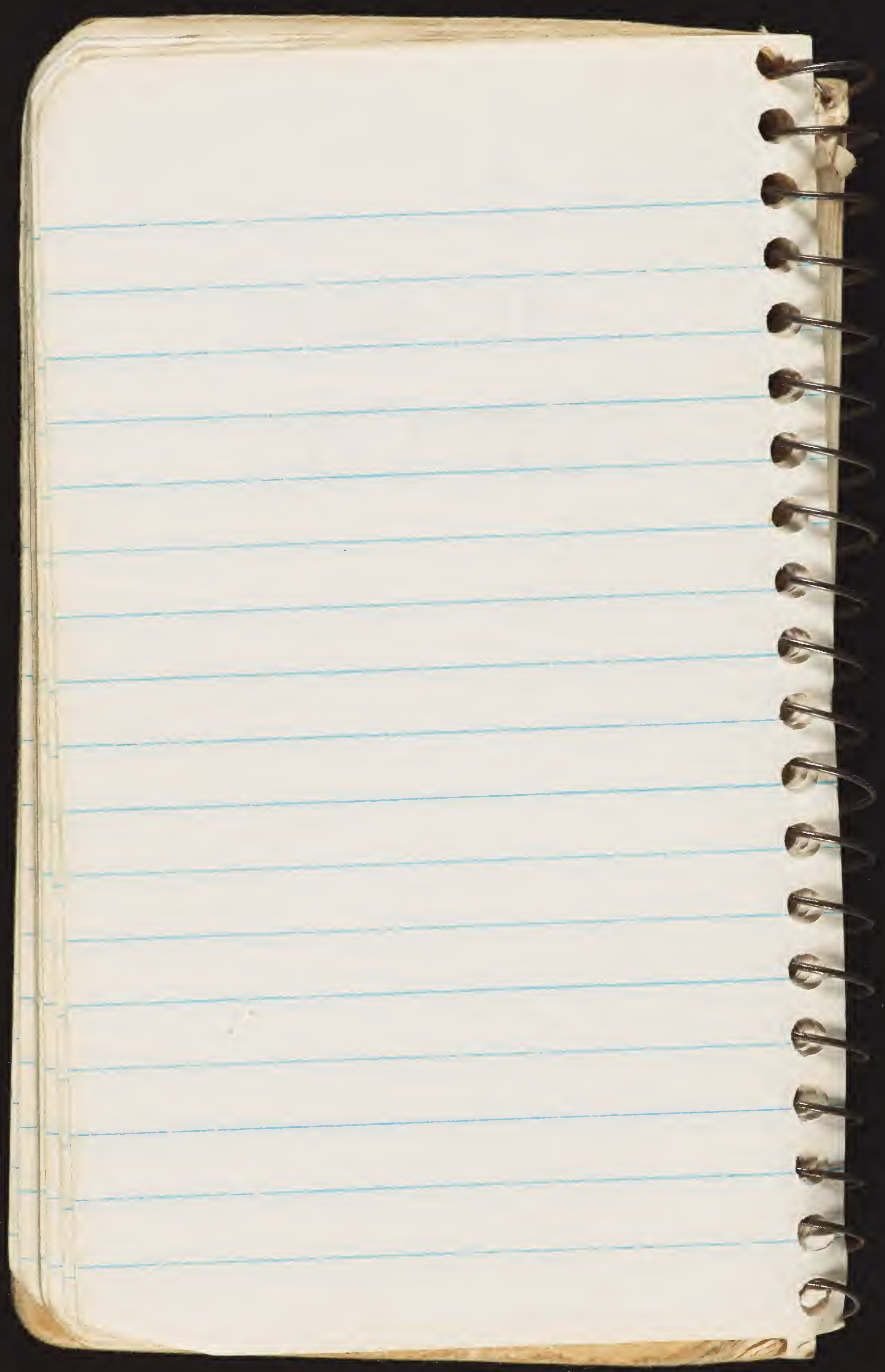




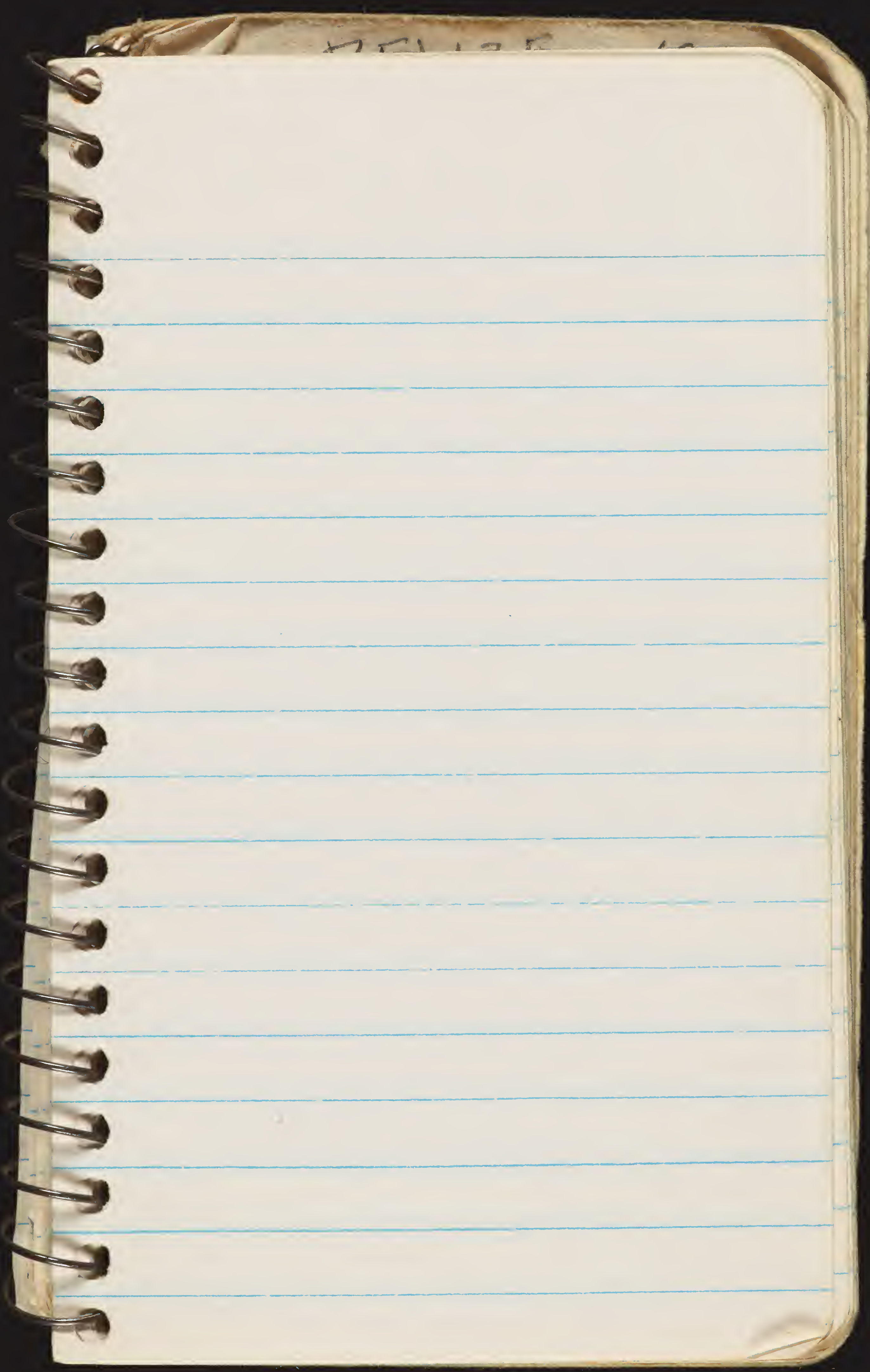





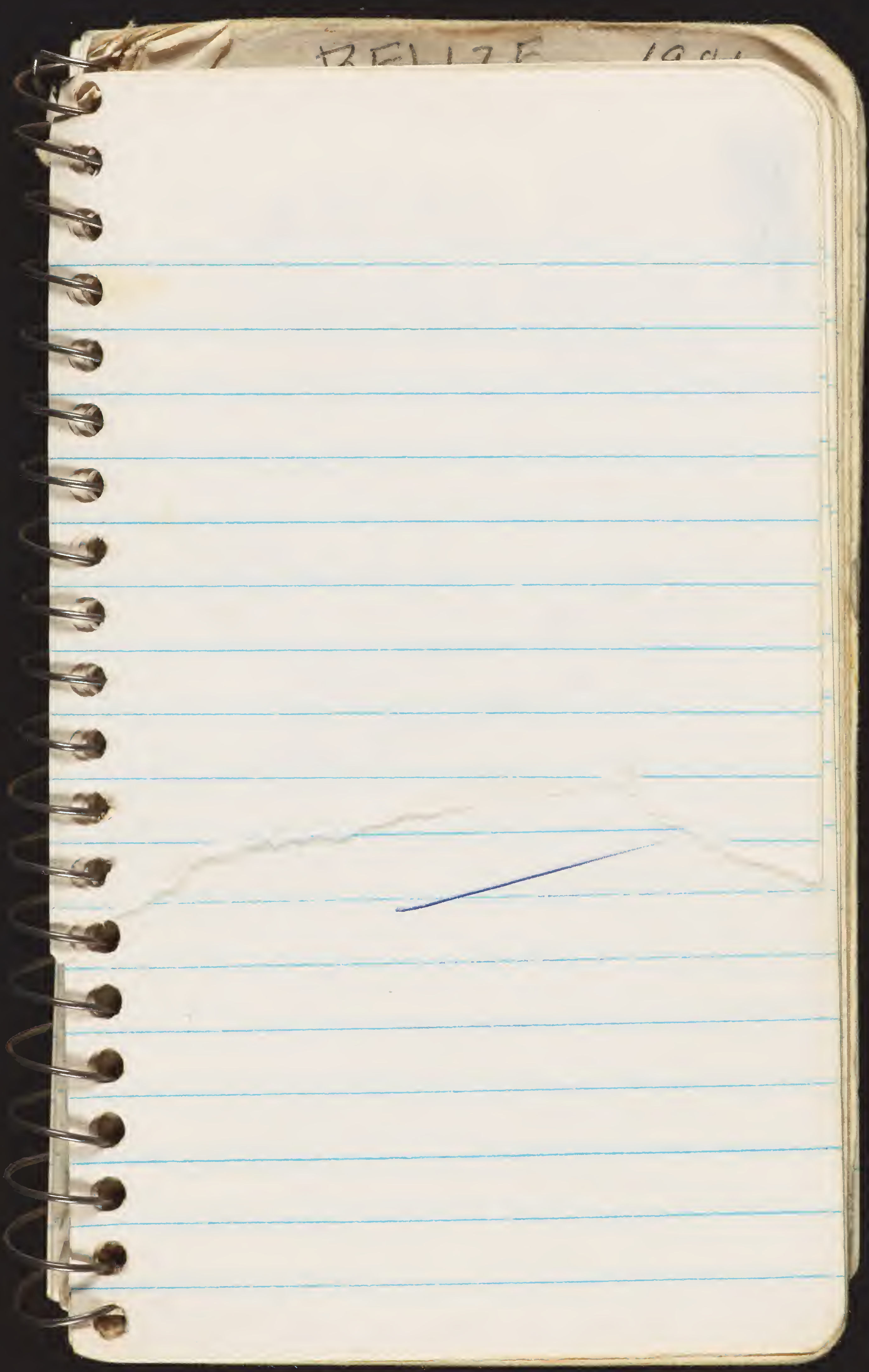



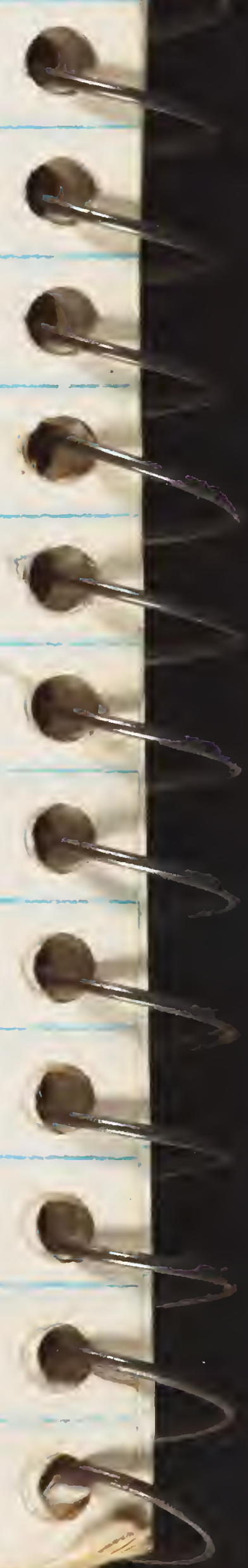

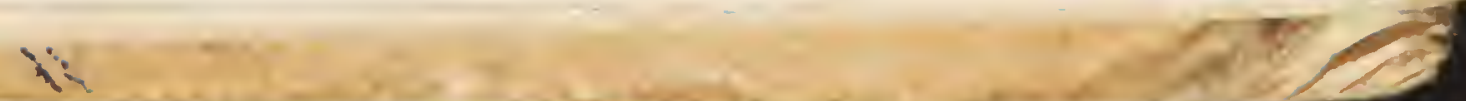


Bill Orberm

Harrey \& Mavira

mude

(apis 3)

Dennere with

the mutdo a fill 



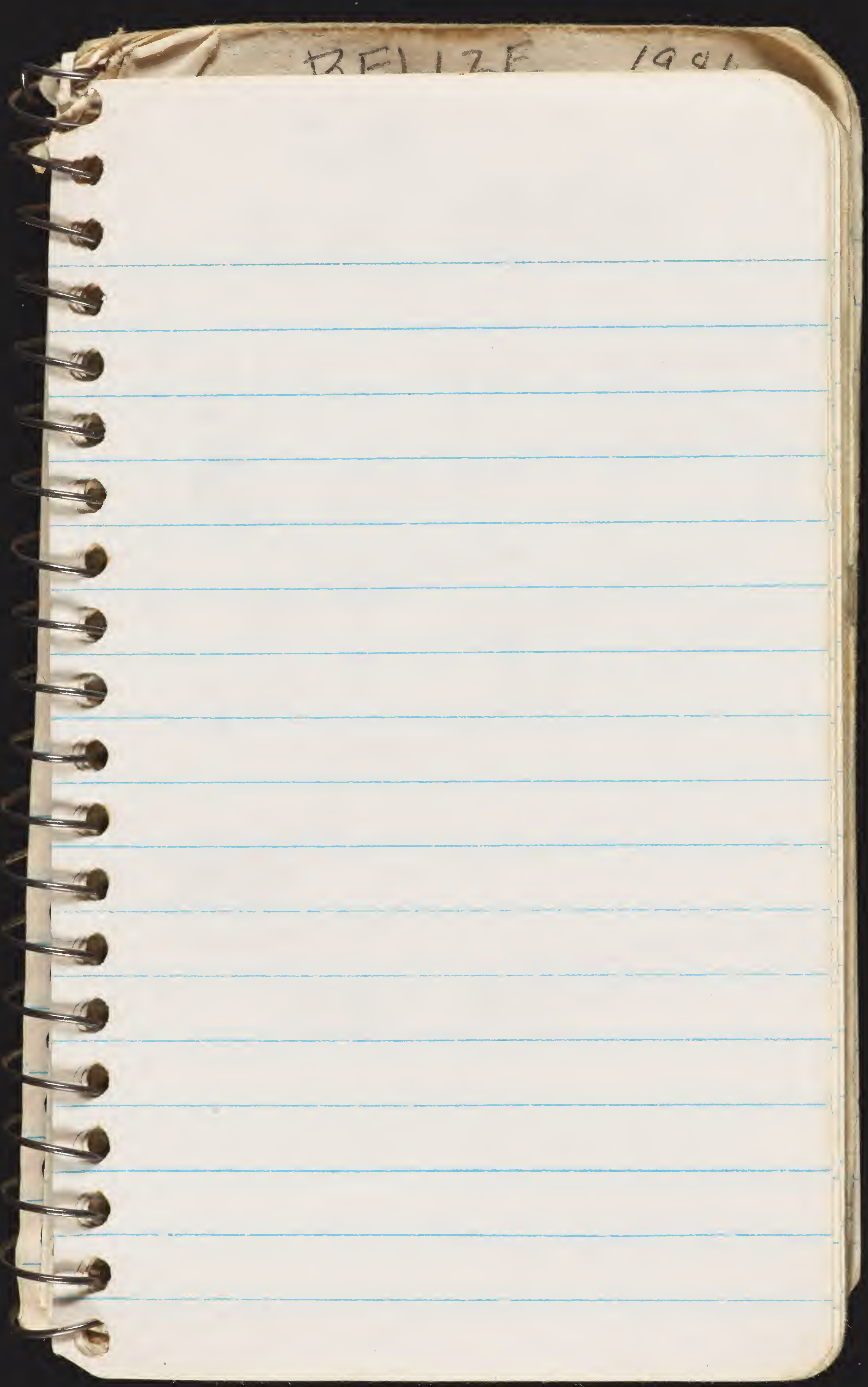








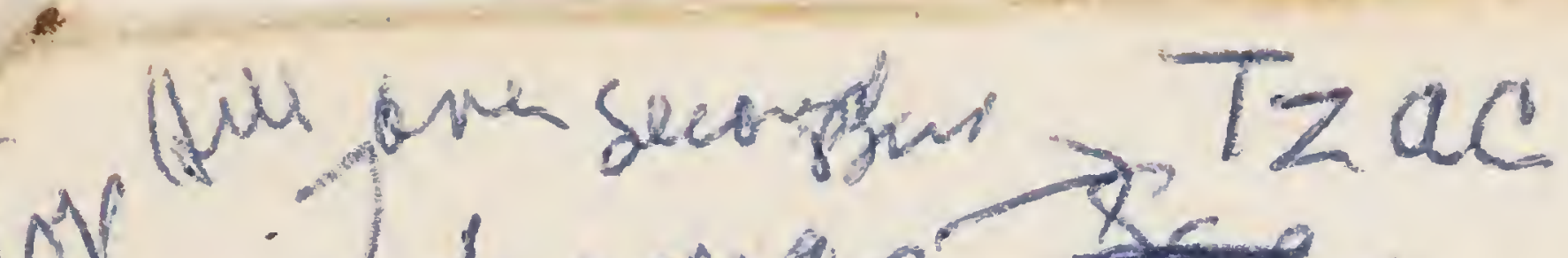

for Llowas - peer silamenre - To Cenráaddo Cho Rigle

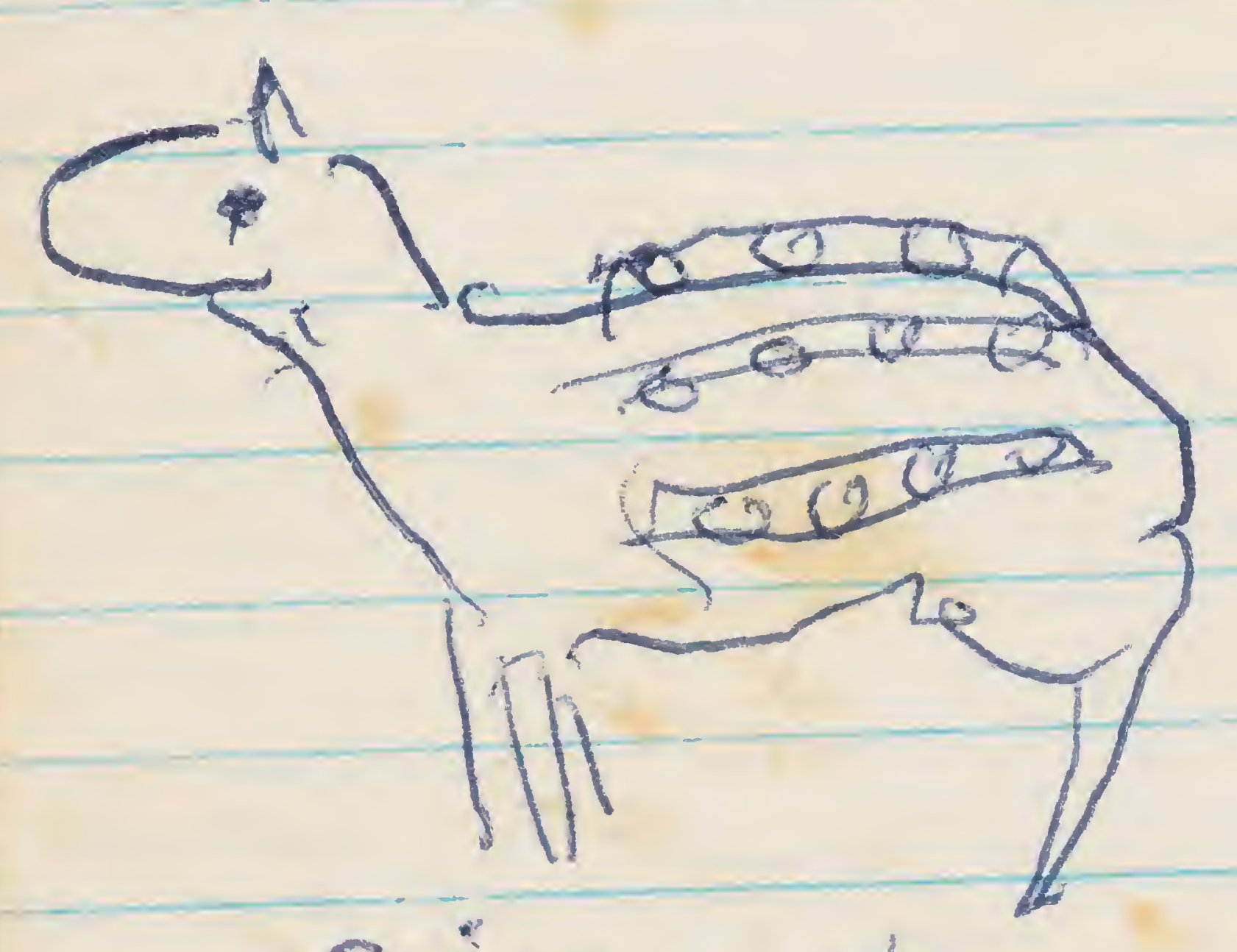

givnot
Paca Labibar

Margant Collins 


\section{BELIZE 1981}




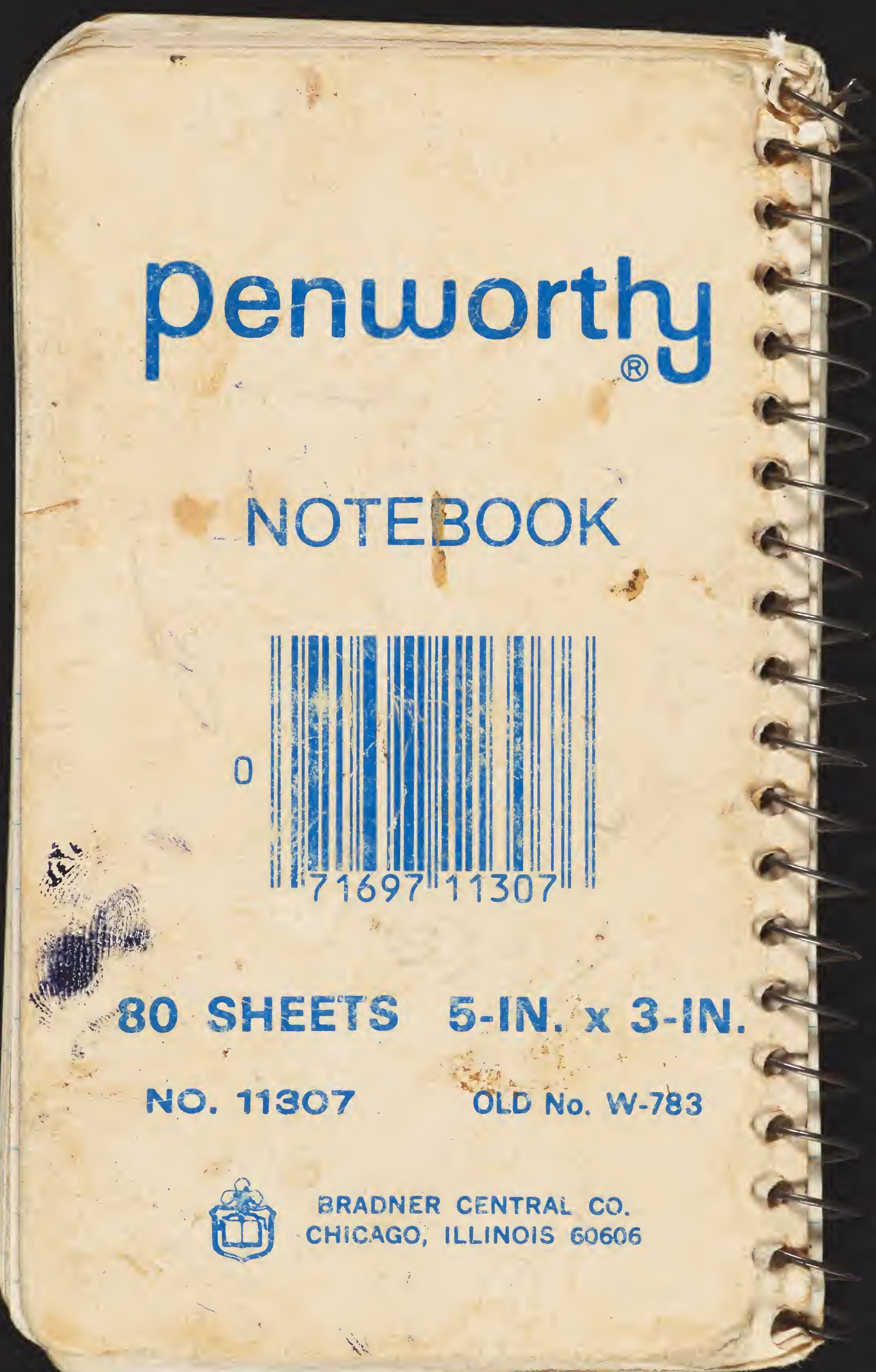


Collected by M.S. Collins unless otherwise indicated

la. Nasutitermes sp. Im. S $W$ from log being used as firewood; June 7, 1981 Blue Creek, Belize - Coll. Tina.

Ib Termitophiles from la $a_{t}$ : in special preservative (Warren steiner) (fuhl Sol.)

2. Amitermes sp., $\mathrm{S}, \mathrm{W}$; deàd branch near building, Blue Creek, June 6, 1981

3. Nasutitermes sp. from dead down branch near building, Blue Creek, June 7 .

4. Amitermes sp. Im., S, W. June 6, 1981-Down branch at Blue Creek, Belize

5. Heterotermes sp. S.W.; June 6, 1981-from dead down branch near building, Blue Creek

6. Heterotermes sp. June 7,1981; From heavily-eaten stump in ground, Blue Creek; $\mathrm{S}, \mathrm{W}$.

7. Combat 1: Large ant running on trail; Heterotermes added, trail disrupted, worke and soldier termites attacked and drove off ants

$\therefore$ Combat 2 - Red army ant vs Heterotermes sp.; ants were diverted from trail, termites attacked and repelled them; ants showed signs of distress after termite contact

- Combat 3 - Red army ant vs Nasutitermes sp.; termites repelled ants effectively; June 8,1981

7. Berds Road, $0.7 \mathrm{mi}$. from junction with Lubantin Rd., Belize June 8

8. Seive colony, Fence post beside road to Blue Creek, $4 \mathrm{mi}$. north of Blue Creek; 200 soldiers to hexane; Nasutitermes sp. ; June 8.

9. $\quad$ from dead branch on ground beside road in second-growth bordering Columbia Road, ca $5 \mathrm{mi}$. from Blue Creek

10.Staphylinidae eating stray termites at site of nest picking, Nasutitermes sp., June 9

11. Hwwittirm $\mathscr{P}$, beside road, second growth, near store, $6 \mathrm{mi}$. from

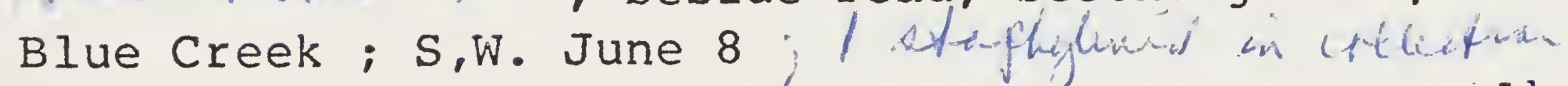

12. June 8 - Beside road, cartón nest on palm tree ca $1 \frac{1}{2} \mathrm{ft}$. above ground; ca $6 \mathrm{mi}$. from Blue Creek, cleared area beside road, second-growth forest; $100 \mathrm{~s}$ in hexane

Combat with Monacis (?) sp. ,photographed at length : Ants captured termites; showed temporary distress after being fired upon, but went on to clean, then attack more termites. Nest material with termites in it placed in midst of combat at 10:55 AM. After I hour: Termites numerous in nest material and in an orderly train to under surface of log - June 9

13. Armitermes sp. from large down log going uphill from lodge, Blue Creek, June 9.

14. June 9 - Log on ground, as in \#13 but further up trail; coll. Marianne Early ;

15. Heterotermes $s p$, from a standing dead stump up trail from Blue Creek lodge latrine ; June 9, 1981

16. Nasutitermes sp. - looks like $\underline{N}$. octopilis, smells like it, acts like it; June 9 ; dead, down HARD stump; $\mathrm{S}, \mathrm{W}$.

Combat with Monacis (?); block of wood containing ca 30 Nasutitermes sp. workers and soldiers placed in path of foraging Monacis; ants were instantly repelled; circled around termites; termite soldiers fired on any ant that approached; group still intact after 30 minutes. Parties separated out leaving wood, later returned. Soldiers slow, but repelled ants effectively. I worker captured at beginning of engagement; another worker captured at 45 minutes combat time. observations terminated at 60 minutes. Surviving termites returned to culture ; 6 sprayed ants isolated to plastic cups. At 24 hours, 3 were alive; 1 was dead; and 2 had escaped.

17. Amitermes sp. S,W. From down, dead wood near lodge at Blue Creek; 10 June, 1981.

18. Nasutitermes sp. large colony in rotten log on trail to falls, Blue Creek; 10 June 1981; 100 to hexane

Combat: 12:30 PM June 10; ca 200 termites from colony placed on Monacis trail; ants seizes workers and soldiers immediately; nearly all carried off by ants within 30 minutes. 